\title{
Tarifpolitischer Halbjahresbericht: Zwischenbilanz der Lohn- und Gehaltsrunde 2008
}

\author{
Reinhard Bispinck \\ WSI-Tarifarchiv
}

Die Tarifabschlüsse im ersten Halbjahr 2008 fallen insgesamt deutlich höher aus als im vergangenen Jahr. Bisher brachten sie den Beschäftigten im Durchschnitt Einkommenszuwächse von 4,6 \%. Darin kommen vor allem die positiven Ergebnisse bei Stahl, Chemie und im öffentlichen Dienst zum Ausdruck. In einigen anderen Branchen sind die Abschlüsse dagegen deutlich niedriger ausgefallen. Berücksichtigt man die noch laufenden Tarifabschlüsse der Vorjahre, so wird gesamtwirtschaftlich immerhin eine kleine tarifliche Reallohnsteigerung erreicht. Die Tariferfolge sind nicht zuletzt das Resultat einer hohen Mobilisierungsfähigkeit der Gewerkschaften und der Bereitschaft der Beschäftigten, sich an Warnstreiks und Arbeitsniederlegungen zu beteiligen.

\section{Einleitung}

Die ökonomischen Rahmenbedingungen der Tarifrunde entwickelten sich zwiespältig. Zum einen erwarteten die Ökonomen infolge der Turbulenzen an den internationalen Finanzmärkten und der nachlassenden globalen Konjunktur auch für Deutschland eine Abschwächung der wirtschaftlichen Entwicklung. Der Sachverständigenrat prognostizierte für 2008 eine Steigerung des Bruttoinlandsprodukts in Höhe von $1,9 \%$, im Jahreswirtschaftsbericht ging die Bundesregierung von 1,7\% aus und das Institut für Makroökonomie und Konjunkturforschung (IMK) reduzierte seine Wachstumsprognose für 2008 sogar auf 1,5\% (SVR 2007; JWB 2007; IMK 2007). Zumindest in den ersten Monaten des Jahres entwickelte sich die Konjunktur in Deutschland jedoch günstiger als erwartet. Auch die Belebung des Arbeitsmarktes hielt zunächst noch an. Für die Tarifrunde entscheidend waren die anhaltend positive Gewinnentwicklung der privaten Wirtschaft, die kräftige Steigerung der Manager- und Vorstandsbezüge und das bei den Beschäftigten ausgeprägte Gefühl, am jahrelangen Aufschwung bislang nicht oder nur unzureichend partizipiert zu haben. Die daraus resultierende Erwartungshaltung fand ihren Niederschlag auch in den Tarifforderungen.

Die Lohn- und Gehaltsforderungen der Gewerkschaften fielen in dieser Tarifrunde noch einmal höher aus als im Vorjahr. An der Spitze lag der öffentliche Dienst. Dort forderte die Vereinte Dienstleistungsgewerkschaft (ver.di) eine Tarifanhebung von

\begin{tabular}{|c|c|}
\hline Bankgewerbe & 8,0 \\
\hline Chemische Industrie & 7,0 \\
\hline Deutsche Post AG & 7,0 \\
\hline Eisen- und Stahlindustrie & 8,0 \\
\hline Kfz-Gewerbe Nordrhein-Westfalen & 5,0 \\
\hline Landwirtschaft & 5,5 \\
\hline Nahrung-Genuss-Gaststätten & $4,5-6,0$ \\
\hline Öffentlicher Dienst (Bund, Gemeinden) & 8,0 (mind. $200 €$ ) \\
\hline Papier verarbeitende Industrie & 8,0 \\
\hline Redakteure (Tageszeitungen und Zeitschriften) & 7,5 \\
\hline Textil- und Bekleidungsindustrie & 5,5 \\
\hline
\end{tabular}

$8 \%$ mindestens jedoch $200 €$. Ebenfalls $8 \%$ forderten die Gewerkschaften u. a. in der Stahlindustrie, in der Papierverarbeitung und im Bankgewerbe. Erstmals seit 2004 stellte die IG BCE für die chemische Industrie mit $7 \%$ wieder eine bezifferte Forderung auf. In anderen Branchen blieben die Forderungen mit 4,5\% bis 5,5\% deutlich darunter (Tabelle 1).

Nach dem Kündigungsterminkalender liefen die Tarifverträge im öffentlichen Dienst Ende Dezember 2007 als erste aus, gefolgt von der Eisen- und Stahlindustrie Ende Januar 2008. Die Entgelttarifverträge in der chemischen Industrie liefen regional unterschiedlich zwischen Ende Februar und Ende April aus. Die Verträge im westdeutschen Kfz-Gewerbe endeten überwiegend ebenfalls Ende Februar.

Den ersten wichtigen Abschluss (Übersicht 1) gab es am 20.2.2008 in der Eisenund Stahlindustrie (Abschnitt 3.1). Für Februar wurde eine Pauschale von $200 €$ gezahlt, zum 1.3.2008 folgte eine Tarifanhebung um 5,2 \% mit einer Laufzeit bis Ende März 2009. Am 31.3.2008 vereinbarten die
Tarifparteien im öffentlichen Dienst (Bund, Gemeinden) einen Sockelbetrag von $50 €$ sowie eine Anhebung um 3,1\% ab dem 1.1.2008 (für die Gemeinden Ost erfolgte beides ab 1.4.2008); es folgt eine Einmalzahlung von $225 €$ zum Jahresbeginn 2009 und einer Stufenerhöhung von 2,8 \% ebenfalls ab dem 1.1.2009 mit einer Laufzeit bis Ende 2009 (Abschnitt 3.2). Am 16.4.2008 erreichte die IG BCE für die chemische Industrie folgenden Abschluss (Abschnitt 3.3): Regional unterschiedlich gibt es ab März/ April/Mai 2008 eine Tarifanhebung von 4,4\%, gefolgt von einer Stufenerhöhung von 3,3\% ab April/Mai/Juni 2009. Für die ersten 13 Monate gibt es eine zusätzliche

Reinhard Bispinck, Dr., Wissenschaftler im WSI und Leiter des WSI-Tarifarchivs. e-mail: Reinhard-Bispinck@boeckler.de. Götz Bauer, Monika Müller, Ulrich Schmidt, Monika Schwacke-Pilger, Andrea Taube und Monika Wiebel sind Sachbearbeiterinnen und Sachbearbeiter im WSI-Tarifarchiv. 
Einmalzahlung von 0,5\%. Die Gesamtlaufzeit beträgt 25 Monate.

In anderen Branchen gelangen ebenfalls Abschlüsse oberhalb der Inflationsrate: In der Landwirtschaft sieht die Bundesempfehlung eine Erhöhung von 3,8 \% ab dem 1.3.2008 und eine Stufenerhöhung von 3,2 \% ab dem 1.2.2009 bei einer Laufzeit bis Ende März 2010 vor. In der Textilund Bekleidungsindustrie wird nach einem Nullmonat und einer Pauschale für April und Mai 2008 in Höhe von $200 €$ eine Tariferhöhung von 3,6 \% ab dem 1.6.2008 gezahlt. Die Laufzeit reicht bis Ende Februar 2009. Bei der Deutschen Post AG sieht der Tarifabschluss für die Monate Mai bis Oktober eine Pauschale von $200 €$ vor, anschließend erhalten die Beschäftigten eine Tariferhöhung von 4,0\% und eine nochmalige Steigerung um 3,0 \% ab dem 1.11. 2009 mit einer Laufzeit bis Ende Juni 2010. In der Energiewirtschaft NRW (GWE-Bereich) steigen die Tarife ab dem 1.7.2008 um 4,0\%, ein Jahr später folgt eine Stufenerhöhung von 3,5 \% bis Ende Juni 2010. In der Papier verarbeitenden Industrie umfasst der Tarifabschluss nach einem Nullmonat eine Tariferhöhung von 3,9\% ab dem 1.5.2008 sowie eine Stufenerhöhung von zusätzlichen $2,9 \% \mathrm{ab}$ dem 1.5.2009 für weitere 12 Monate.

Geringere Tariferhöhungen wurden vereinbart im Brauereigewerbe NordrheinWestfalen mit einem Plus von 3,0\% für das Jahr 2008. Im Hotel- und Gaststättengewerbe Nordrhein-Westfalen beträgt die Tariferhöhung ebenfalls 3,0 \% ab 1.3.2008, ein Jahr später folgt eine Stufenerhöhung um 2,5\% mit einer Laufzeit bis Ende Mai 2010.

Im Kfz-Gewerbe gelang der erste regionale Abschluss in Hessen: Dort erhalten die Beschäftigten nach einem Nullmonat $\mathrm{ab}$ dem 1.4.2008 eine Tariferhöhung von 2,5 \%, ab dem 1.12.2008 eine Stufenerhöhung von $2,0 \%$ und ein Jahr später weitere 2,0 \%. Die Laufzeit reicht bis Ende April 2010. Ähnliche Abschlüsse gab es auch in anderen regionalen Tarifbereichen. In Nordrhein-Westfalen ließ der zuständige Verband die Verhandlungen mit der IG Metall kurz vor der endgültigen Einigung platzen und gab sein tarifpolitisches Mandat ganz auf. Kurz zuvor hatte der Verband mit der konkurrierenden „Christlichen Gewerkschaft Metall“ einen Tarifvertrag zu deutlich schlechteren Konditionen abgeschlossen. Die IG Metall versucht nun, über Firmentarifverträge schrittweise die Tarif-

\begin{tabular}{|c|c|c|}
\hline \multicolumn{3}{|c|}{$\begin{array}{l}\text { Übersicht 1: Ausgewählte Lohn- und Gehaltsabschlüsse West und Ost } \\
\text { für } 2008\end{array}$} \\
\hline Abschluss & Tarifbereich & Ergebnis \\
\hline 23.01.2008 & Landwirtschaft & $\begin{array}{l}\text { Bundesempfehlung: } \\
\mathbf{3 , 8} \% \text { ab 01.03.08 } \\
\mathbf{3 , 3} \% \text { Stufenerhöhung ab 01.02.09, Laufzeit bis } 31.03 .10\end{array}$ \\
\hline 11.02.2008 & $\begin{array}{l}\text { Brauereien Nordrhein- } \\
\text { Westfalen }\end{array}$ & $3,0 \%$ ab 01.01.08, Laufzeit bis 31.12 .08 \\
\hline 20.02.2008 & $\begin{array}{l}\text { Eisen- und Stahlindustrie } \\
\text { Niedersachsen, Bremen, } \\
\text { Nordrhein-Westfalen }\end{array}$ & $\begin{array}{l}200 € \text { Pauschale für Februar } 2008 \\
5,2 \% \text { ab 01.03.08, Laufzeit bis } 31.03 .09\end{array}$ \\
\hline 21.02 .2008 & $\begin{array}{l}\text { Hotel- und } \\
\text { Gaststättengewerbe } \\
\text { Nordrhein-Westfalen }\end{array}$ & $\begin{array}{l}3,0 \% \text { ab 01.03.08 } \\
2,5 \% \text { Stufenerhöhung ab 01.03.09, Laufzeit bis } 31.05 .10\end{array}$ \\
\hline 07.03 .2008 & Kfz-Gewerbe Hessen & $\begin{array}{l}\text { Nach } 1 \text { Nullmonat (März) } \\
2,5 \% \text { ab 01.04.08 } \\
2,0 \% \text { Stufenerhöhung ab 01.12.08 } \\
2,0 \% \text { Stufenerhöhung ab 01.12.09, Laufzeit bis } 30.04 .10\end{array}$ \\
\hline 11.03 .2008 & $\begin{array}{l}\text { Textil- und Bekleidungs- } \\
\text { industrie West }\end{array}$ & $\begin{array}{l}\text { Nach } 1 \text { Nullmonat (März) } \\
200 € \text { Pauschale insg. für April und Mai } 2008 \\
3,6 \% \text { ab 01.06.08, Laufzeit bis } 28.02 .09\end{array}$ \\
\hline 31.03.2008 & $\begin{array}{l}\text { Öffentlicher Dienst } \\
\text { Bund, Gemeinden } \\
\text { West und Ost }\end{array}$ & $\begin{array}{l}50 € \text { Sockelbetrag plus } \\
3,1 \% \text { ab 01.01.08 (Gemeinden Ost: beides ab 01.04.08) } \\
2,8 \% \text { Stufenerhöhung ab 01.01.09 } \\
225 € \text { Einmalzahlung zum 01.01.09, Laufzeit bis } 31.12 .09 \\
\text { (Abweichungen bei Krankenhäusern, Nahverkehr und } \\
\text { Versorgung) }\end{array}$ \\
\hline 03.04 .2008 & $\begin{array}{l}\text { Einzelhandel: } \\
\text { REWE-Gruppe }\end{array}$ & $\begin{array}{l}50 € \text { Pauschale für jeden tariflosen Monat in } 2007 \\
3,0 \% \text { ab 01.01.08, Laufzeit bis } 30.04 .09\end{array}$ \\
\hline 08.04 .2008 & $\begin{array}{l}\text { Ärzte/innen an kommu- } \\
\text { nalen Krankenhäusern } \\
\text { (Marburger Bund) }\end{array}$ & $\begin{array}{l}\text { 4,0 \% durchschnittlich ab 01.04.08 } \\
\mathbf{3 , 8} \% \text { Stufenerhöhung ab 01.01.09, Laufzeit bis } 31.12 .09\end{array}$ \\
\hline 16.04 .2008 & Chemische Industrie & $\begin{array}{l}4,4 \% \text { regional unterschiedlich ab 03/04/05/2008 } \\
3,3 \% \text { Stufenerhöhung ab 04/05/06/2009, Laufzeit bis } \\
03 / 04 / 05 / 2010 \\
\text { Einmalzahlung von 0,5 \% für die ersten } 13 \text { Monate }\end{array}$ \\
\hline 22.04 .2008 & Steinkohlenbergbau & $\begin{array}{l}300 € \text { Pauschale insg. für Januar-April } \\
3,4 \% \text { ab 01.05.08 } \\
2,0 \% \text { Stufenerhöhung ab 01.07.09, Laufzeit bis } 31.12 .10\end{array}$ \\
\hline 30.04 .2008 & Deutsche Post AG & $\begin{array}{l}200 € \text { Pauschale insg. für Mai-Oktober } \\
4,0 \% \text { ab 01.11.08 } \\
3,0 \% \text { Stufenerhöhung ab 01.12.09, Laufzeit bis } 30.06 .10\end{array}$ \\
\hline 08.05 .2008 & Papierverarbeitung & $\begin{array}{l}\text { Nach } 1 \text { Nullmonat (April) } \\
3,9 \% \text { ab 01.05.08 } \\
\mathbf{2 , 9} \% \text { Stufenerhöhung ab 01.05.09, Laufzeit bis } 30.04 .10\end{array}$ \\
\hline 29.05.2008 & $\begin{array}{l}\text { Energiewirtschaft } \\
\text { Nordrhein-Westfalen } \\
\text { (GWE) }\end{array}$ & $\begin{array}{l}\mathbf{4 , 0} \% \text { ab 01.07.08 } \\
\mathbf{3 , 5} \% \text { Stufenerhöhung ab 01.07.09, Laufzeit bis } 30.06 .10\end{array}$ \\
\hline & rchiv, Stand: Juni 2008. & WSI mitTEILUNGEN \\
\hline
\end{tabular}

bindung und perspektivisch auch den Flächentarifvertrag wiederherzustellen.

Im Einzelhandel hat ver.di nach den seit rund einem Jahr erfolglosen regionalen Verhandlungen am 3.4.2008 einen Tarifvertrag mit der REWE-Gruppe abgeschlossen. Er sieht eine Pauschalzahlung von $50 €$ für jeden tariflosen Monat im Jahr 2007 sowie eine Tarifanhebung um 3,0\% ab 1.1.2008 mit einer Laufzeit bis Ende April 2009 vor. Auch eine Regelung für die umstrittenen Spätarbeitszuschläge wurde gefunden: Es wurde eine zusätzliche variable Jahressondervergütung in Höhe von 12,5 \% $(0-25 \%)$ des individuellen monatlichen Tarifentgelts vereinbart. Im Gegenzug fallen die Samstagszuschläge bis $18.30 \mathrm{~h}$ weg. Erstmals werden alle Samstage, auch die vier vor Weihnachten, ab $18.30 \mathrm{~h}$ zuschlags- pflichtig. Die Zuschlagregelung von Montag bis Freitag ab 18.30 h sowie die Nachtarbeitszuschläge bleiben unverändert. Ver.di gelang es erst nach 15 Monaten, einen ersten regionalen Abschluss durchzusetzen (Abschnitt 3.4).

\section{Ergebnisse im Überblick}

Im ersten Halbjahr des Jahres 2008 schlossen die Gewerkschaften des DGB Einkommenstarifverträge für rund 4,4 Mio. Beschäftigte ab, darunter rund 0,7 Mio. in den neuen Bundesländern. Das entspricht einem knappen Viertel der von Tarifverträgen erfassten Beschäftigten. 


\begin{tabular}{lccc}
\hline Tabelle 2: Tarifsteigerung $\mathbf{2 0 0 8}^{1}$ - in \% - & & & \\
\hline Wirtschaftsbereich & West & Ost & Gesamt \\
\hline Gartenbau, Land- und Forstwirtschaft & 3,4 & 3,9 & 3,7 \\
Energie- und Wasserversorgung, Bergbau & 2,9 & 3,5 & 3,0 \\
Grundstoff- und Produktionsgütergewerbe & 3,3 & 4,0 & 3,4 \\
Investitionsgütergewerbe & 2,2 & 2,0 & 2,2 \\
Verbrauchsgütergewerbe & 3,0 & 2,7 & 2,9 \\
Nahrungs- und Genussmittelgewerbe & 2,5 & 2,8 & 2,5 \\
Baugewerbe & 3,0 & 3,0 & 3,0 \\
Handel & 3,1 & 3,1 & 3,1 \\
Verkehr und Nachrichtenübermittlung & 4,2 & 4,3 & 4,2 \\
Kreditinstitute, Versicherungsgewerbe & 2,7 & 2,7 & 2,7 \\
Private Dienstleistungen, Org. o. Erwerbszweck & 2,9 & 2,9 & 2,9 \\
Gebietskörperschaften, Sozialversicherung & 3,1 & 8,7 & 4,3 \\
\hline Gesamte Wirtschaft & 3,0 & $\mathbf{5 , 0}$ & $\mathbf{3 , 3}$ \\
\hline 1) Jahresbezogene Erhöhung 2008 gegenüber 2007. & & W/SI MITTEILUNGEN \\
Quelle: WSI-Tarifarchiv 2008, Stand: 15.6.2008. & & &
\end{tabular}

Die durchschnittliche Abschlussrate betrug für die Beschäftigten in Westdeutschland 6,5\%, für die Beschäftigten in Ostdeutschland 8,5\%. Diese Größe berücksichtigt nur die tabellenwirksamen Tarifanhebungen und schließt auch weitere Stufenanhebungen ein, die während der Laufzeit der Verträge wirksam werden. Pauschal- und zusätzliche Einmalzahlungen werden hingegen nicht berücksichtigt.

Berücksichtigt man ausschließlich die im Jahr 2008 in Kraft tretenden Tariferhöhungen, ergibt sich eine Steigerung von 4,6\% (West: 4,3\%, Ost: 6,0\%). Differenziert man diese Größe nach Wirtschaftsbereichen, dann ergibt sich in diesem Jahr folgende Streuung: Am unteren Ende liegen das Nahrungs- und Genussmittelgewerbe mit 3,0 \% sowie die Bereiche Investitionsgütergewerbe, Verbrauchsgütergewerbe und Private Dienstleistungen, Organisationen ohne Erwerbszweck mit je 3,7 \%. An der Spitze stehen der Bereich Gebietskörperschaften, Sozialversicherungen mit 5,6\% sowie das Grundstoff- und Produktionsgütergewerbe mit $4,6 \%$.

In Relation $\mathrm{zu}$ anderen gesamtwirtschaftlichen Größen ist die jahresbezogene Steigerung der Tarifverdienste heranzuziehen (Tabelle 2). Diese Größe bringt - anders als die Abschlussrate - die Steigerung des durchschnittlichen tariflichen Monatsentgelts des laufenden Jahres gegenüber dem Vorjahr zum Ausdruck. Einbezogen werden hier auch die Pauschalzahlungen, z. B. für verzögert in Kraft getretene Neuabschlüsse und zusätzliche Einmalzahlungen. Auf der Basis der vorliegenden Abschlüsse (Stichtag 15.6.2008) beträgt die kalenderjährliche Erhöhung der Tarifverdienste für dieses Jahr durchschnittlich 3,6\%. Für jene Wirtschaftszweige und Ta- rifbereiche, für die bereits im vergangenen Jahr oder früher Tarifanhebungen für das gesamte Jahr 2008 vereinbart wurden, errechnet sich eine jahresbezogene Steigerung von $3,1 \%$. Insgesamt ergibt sich für 9,0 Mio. erfasste Beschäftigte eine durchschnittliche Tariferhöhung für das Jahr 2008 von $3,3 \%$.

Die kalenderjährliche Tarifsteigerung liegt mit 3,3\% in diesem Jahr voraussichtlich gut einen Prozentpunkt über dem Wert ner laufenden Preissteigerungsrate von rund $3 \%$ wird mit dieser Tariferhöhung immerhin gesamtwirtschaftlich eine kleine tarifliche Reallohnsteigerung erreicht. $\mathrm{Zu}$ berücksichtigen ist allerdings, dass große Tarifbereiche, wie z. B. der Einzelhandel mit rund 2 Mio. Beschäftigten, darin noch nicht enthalten sind. Auch die Metall- und Elektroindustrie mit rund 3,4 Mio. Beschäftigten ist noch nicht berücksichtigt. Dort laufen die Tarifverträge erst Ende Oktober dieses Jahres aus.

Zwischen den einzelnen Wirtschaftsbereichen ergeben sich deutliche Unterschiede. Die Spannweite zwischen ihnen fällt allerdings in diesem Jahr geringer aus als im Vorjahr. Sie variiert zwischen 2,2 \% im Investitionsgütergewerbe und 4,3\% im Bereich Gebietskörperschaften, Sozialversicherung. Dazwischen liegen u.a. die Bereiche Verkehr und Nachrichtenübermittlung mit 4,2 \%, Gartenbau, Land- und Forstwirtschaft mit 3,7 \%, Grundstoff- und Produktionsgütergewerbe mit 3,4\%, Baugewerbe mit 3,0 \% sowie Private Dienstleistungen, Organisationen ohne Erwerbszweck mit 2,9\%.

Ein Blick auf das Tarifniveau Ost/West, d. h. das Verhältnis der tariflichen Grundvergütungen in den neuen im Vergleich zu des Vorjahres (2007: 2,2 \%). Angesichts ei- den alten Ländern, ergibt am Beispiel ausgewählter Tarifbereiche folgendes Bild: Im Durchschnitt von rund 40 ausgewählten Tarifbereichen mit rund 1,8 Mio. Beschäftigten errechnet sich gegenüber Ende 2007 ein Anstieg des Tarifniveaus Ost/West von 95,2 auf 96,7 \%. Die Ursache für den deutlichen Anstieg liegt vor allem in der Tarifniveauanhebung im öffentlichen Dienst.

\section{Ausgewählte Tarifrunden}

\subsection{STAHLINDUSTRIE}

Bereits zum dritten Mal in Folge konnte in der Stahlindustrie ein tarifpolitisches Ergebnis vereinbart werden, das sich deutlich aus dem tarifpolitischen Umfeld abhebt. Der vorangegangene Abschluss vom September 2006 hatte eine Pauschalzahlung von $500 €$, eine Tarifanhebung von 3,8\% und eine zusätzliche Einmalzahlung von $750 €$ beinhaltet. Er lief Ende Januar 2008 aus. Angesichts der weiterhin hervorragenden Branchenkonjunktur (IG Metall 2007) stellte die IG Metall im Dezember 2007 für die Stahlindustrie in Niedersachsen, Bremen und Nordrhein-Westfalen sowie Ostdeutschland folgende Tarifforderungen auf, die sie unter die Überschrift „Mehr Geld und humanere Arbeitsbedingungen “ stellte:

- Erhöhung der tariflichen Löhne und Gehälter um 8,0 \% ab Februar 2008 mit einer Laufzeit von zwölf Monaten;

- Anhebung der Ausbildungsvergütungen um $100 €$; sie waren bei den Tarifrunden 2005 und 2006 aufgrund eines zusätzlichen Angebotes von Ausbildungsplätzen nicht erhöht worden;

- Einführung eines zusätzlichen freien Arbeitstages pro Kalenderjahr für Beschäftigte ab dem 50. Lebensjahr unter Fortzahlung des regelmäßigen Arbeitsverdienstes;

- weitere Verhandlungen zu einem „Gemeinsamen Entgeltrahmentarifvertrag (GERT)“.

In der ersten Verhandlung am 21.1.2008 für die westdeutschen Tarifgebiete stießen die gewerkschaftlichen Forderungen erwartungsgemäß auf scharfe Kritik der Stahlarbeitgeber. Zwar räumten sie eine anhaltende Sonderkonjunktur ein, betonten aber die wachsenden Risiken des Stahlmarktes 
und stellten heraus, dass die Beschäftigten bereits mit Sondervergütungen an den steigenden Gewinnen beteiligt würden. Die IG Metall machte frühzeitig Druck auf die Arbeitgeber. Nachdem auch die zweite Verhandlungsrunde am 29.1. ohne Arbeitgeberangebot blieb, begann sie unmittelbar nach Ende der Friedenspflicht am 30.1. mit Warnstreiks. Bis zur nächsten Verhandlungsrunde nahmen über 16.000 Beschäftigte aus 58 Betrieben der Stahlindustrie an Warnstreiks mit einer Dauer von bis zu acht Stunden teil. Die IG Metall kündigte außerdem bereits für Mitte Februar eine Urabstimmung und Streiks an, falls es bis dahin nicht zu einem akzeptablen Tarifergebnis komme. Die Arbeitgeber legten daraufhin am 12.2. ein erstes Angebot vor: Danach sollten die Löhne und Gehälter um 3,5\% angehoben werden bei einer Laufzeit von 16 Monaten bis Ende Mai 2009. Die IG Metall lehnte das Angebot ab, weil es viel zu niedrig, in der Laufzeit deutlich zu lang und damit in Gänze inakzeptabel sei. Damit - so die Einschätzung auf Gewerkschaftsseite - provozierten die Arbeitgeber nur weitere massive Warnstreiks. Tatsächlich startete die Gewerkschaft eine zweite Warnstreikwelle, an der sich mehr als 20.000 Beschäftigte beteiligten. Insgesamt lag die Beteiligung nach Gewerkschaftsangaben mit knapp 40.000 Beschäftigten doppelt so hoch wie im Jahr 2006. Am 19.2. fand die vierte Verhandlungsrunde statt, die mit folgendem Ergebnis endete, das am 21.2. auch für die ostdeutsche Stahlindustrie übernommen wurde:

- $200 €$ Pauschale für Februar 2008;

- Erhöhung der Tariflöhne und -gehälter um 5,2\% ab dem 1.3.2008;

- Erhöhung der Ausbildungsvergütungen in allen Ausbildungsjahren um jeweils $70 €$;

- Laufzeit von insgesamt 14 Monaten bis zum 31.3.2009;

- Vereinbarung über den Abschluss der GERT-Verhandlungen bis Ende Juni 2009.

Eine Verkürzung der Arbeitszeit für Ältere konnte die IG Metall nicht durchsetzen. Materiell bewertete die Gewerkschaft den Abschluss sehr positiv, weil es sich um das beste Ergebnis seit 15 Jahren handele. Tatsächlich wurde der letzte höhere Abschluss im Jahr 1991 vereinbart. Die Tarifkommission stimmte dem Ergebnis daher auch einstimmig zu. Die Stahlarbeitgeber waren weniger zufrieden. Ihr Vorsitzender
Helmut F. Koch machte deutlich, dass vor allem der angedrohte Streik zu diesem Ergebnis geführt habe. Er hätte „gravierende Auswirkungen" gehabt, die man habe vermeiden wollen. Weitere Verhandlungen seien jedoch "nicht erfolgversprechend“ gewesen. Für die Bundesvereinigung der Deutschen Arbeitgeberverbände (BDA) warnte deren Präsident Dieter Hundt, dass dieser Abschluss „auf keinen Fall“ auf andere Branchen übertragen werden dürfe (BDA-Pressemeldung vom 20.2.2008). Gleichwohl war die "Stahl-Vorlage“ (FAZ vom 21.2.2008) in der Welt und entfaltete ihre zumindest mittelbare Orientierungswirkung.

\section{2 ÖFFENTLICHER DIENST}

Im öffentlichen Dienst gelang es den Gewerkschaften erstmals nach drei Jahren, wieder Anschluss an die allgemeine Einkommensentwicklung zu gewinnen. Durch eine sehr hohe Mobilisierung der Mitglieder, die ihren Ausdruck in zwei massiven Warnstreikwellen fand, konnten die kooperierenden Gewerkschaften des öffentlichen Dienstes des DGB wie auch der dbb tarifunion soviel Druck entfalten, dass ein für viele Beobachter überraschend hohes materielles Ergebnis erzielt werden konnte. Allerdings mussten die Gewerkschaften bei der Arbeitszeit Zugeständnisse machen.

\section{AUSGANGSSITUATION UND FORDERUNGEN}

Im Februar 2005 war der neue Tarifvertrag für den öffentlichen Dienst (TVöD) abgeschlossen worden, der im Kern eine Zusammenführung und völlige Neugestaltung der bis dahin getrennten Lohn- und Gehaltstarifverträge vorsah (Bispinck/WSITarifarchiv 2006; Meerkamp 2008). Dieser Vertrag gilt für Bund und Gemeinden. Im Mai 2006 schloss ver.di mit den Ländern nach heftigen Auseinandersetzungen einen vergleichbaren Tarifvertrag (TV-L) ab. Mit dem TVöD wurde auch die Entgeltentwicklung der kommenden drei Jahre festgelegt. Vereinbart wurden Pauschalzahlungen von jeweils $300 €$ für die Jahre 2005/ 2006/2007 mit einer Laufzeit bis zum 31.12.2007. Für die Länder wurden ebenfalls Pauschalzahlungen für die Jahre 2006 und 2007 sowie eine Tarifanhebung von 2,9 \% für das Jahr 2008 vereinbart.

Im TVöD waren verschiedene Punkte offen geblieben, für die Übergangsregelun- gen vereinbart worden waren bzw. die erst nach Vertragsabschluss geregelt werden sollten (ver.di 2007). Dazu zählte neben der befristeten Fortführung von Bewährungsund Zeitaufstiegen vor allem die Erarbeitung einer neuen Entgeltordnung, die eine unmittelbare Eingruppierung der Beschäftigten auf Basis der neuen Entgeltgruppen ermöglichen soll. ${ }^{1}$ Aufgrund zahlreicher Verzögerungen, für die ver.di die Arbeitgeberseite verantwortlich machte, rückte die Tarifrunde 2008 näher, ohne dass die sogenannten „Restanten“ geklärt oder gar die neue Entgeltordnung verhandelt worden wären.

Bereits bei der Tarifauseinandersetzung 2006 hatte ver.di mit der dbb tarifunion, dem Zusammenschluss der Gewerkschaften des öffentlichen Dienstes im Deutschen Beamtenbund, eng kooperiert. Nach den dort gesammelten positiven Erfahrungen wurde für die Tarifrunde 2008 im öffentlichen Dienst eine offizielle tarifpolitische Kooperation beschlossen. Sie sah vor, dass die Verhandlungen mit der Arbeitgeberseite gemeinsam geführt werden, wobei ver.di die Federführung haben sollte. Im Übrigen waren wie immer auch die anderen DGBGewerkschaften im Bereich des öffentlichen Dienstes, die GEW sowie die GdP, an den Verhandlungen beteiligt.

Im Vergleich zur allgemeinen Tarifund Einkommensentwicklung waren die Einkommen der Beschäftigten des öffentlichen Dienstes in den vergangenen Jahren deutlich zurückgeblieben und hatten so zur gespaltenen Einkommensentwicklung beigetragen. Während beispielsweise in der Metallindustrie die Tarifeinkommen von 2000 bis 2007 nominal um fast $20 \%$ gestiegen waren, blieben die Tarifentgelte im öffentlichen Dienst in diesem Zeitraum mit einem Plus von knapp über $11 \%$ erheblich darunter. In den Jahren von 2005 bis 2007 mussten die Beschäftigten des öffentlichen Dienstes infolge der geringen Pauschalzahlungen jeweils Realeinkommenseinbußen hinnehmen. Entsprechend hoch waren die Erwartungen für die Entgeltrunde 2008. In den regionalen Vordiskussionen innerhalb von ver.di waren zum Teil Forderungen von bis zu $10 \%$ aufgestellt worden.

\footnotetext{
1 Bislang werden die Beschäftigten noch nach den alten Vorschriften eingruppiert und erst in einem zweiten Schritt den neuen Entgeltgruppen zugeordnet.
} 
Bereits im Vorfeld der Verhandlungen hatte die Vereinigung der kommunalen Arbeitgeberverbände (VKA) mit „10 Punkten zur Tarifrunde 2008“ (VKA 2007) ihre Position abgesteckt. Darin betonten sie das Ziel, die Kommunen und deren Unternehmen im Wettbewerb zu stärken, das kommunale Dienstrecht weiter zu modernisieren und die Sicherheit der Arbeitsplätze zu erhalten. Die Beschäftigten sollten an der allgemeinen Lohnentwicklung angemessen beteiligt werden, die hohen Abschlüsse der Privatwirtschaft könnten jedoch kein Maßstab sein. Die Wochenarbeitszeit sollte wieder einheitlich auf 40 Stunden verlängert und die Leistungsbezahlung ausgeweitet werden. In Krankenhäusern sei kein Spielraum für lineare Tariferhöhungen, solange die Budgetdeckelung nicht aufgehoben sei.

Am 18./19.12.2007 beriet und beschloss die ver.di-Bundestarifkommission für den öffentlichen Dienst folgende Forderungen für die Tarifrunde 2008:

- Lineare Erhöhung der Tarifentgelte um $8,0 \%$, mindestens $200 €$, bei einer Laufzeit von zwölf Monaten;

- Auszubildende: tabellenwirksamer Festbetrag von $120 €$, Ost-West-Angleichung der Ausbildungsvergütung;

- Übernahme der Auszubildenden und zwar unbefristet, in Vollzeit, ausbildungsgerecht und wohnortnah;

- Im Bereich Versorgung (TV-V): Entgelterhöhungen von 9,0 \% sowie Erhöhung der Wechselschicht- und Schichtzulage auf $225 €$ bzw. $150 €$;

- Zeit- und inhaltsgleiche Übertragung des Tarifergebnisses auf den Bereich der Beamten.

Die öffentlichen Arbeitgeber wiesen die Forderung vor allem mit dem Hinweis auf die nicht tragbaren Kosten zurück. Die haushaltspolitischen Möglichkeiten des Bundes seien zu berücksichtigen und das Konsolidierungsziel dürfe nicht gefährdet werden. Die Sockelforderung führe zu einer Erhöhung in den unteren Entgeltgruppen von bis zu $15,5 \%$, die $120 €$ für die Auszubildenden entsprächen einer Erhöhung um bis zu $20 \%$.

\section{VERHANDLUNGEN}

Am 10./11. Januar begannen die Verhandlungen in Potsdam. Zunächst wurden in einem Spitzengespräch in kleinem Kreis die
Grundsatzpositionen ausgetauscht. In der zweiten Verhandlungsrunde am 24.1. präsentierten die Arbeitgeber ein erstes Angebot. Es sah eine Entgelterhöhung vor in Höhe von:

- 2,5\% zum 1.2.2008;

- $1,0 \%$ zum 1.10 .2008 und

- 0,5\% zum 1.3.2009.

Außerdem sollte die Wochenarbeitszeit

- ab 1.7.2008 auf 39,5 Stunden und

- ab 1.1.2009 auf 40 Stunden

verlängert werden.

Die Gewerkschaften wiesen dieses Angebot als Provokation zurück. Das Entgeltangebot sei bei einer Laufzeit von 24 Monaten viel zu gering, außerdem sollten die Beschäftigten es mit einer Arbeitzeitverlängerung größtenteils selbst finanzieren. Die angeblichen $5 \%$ würden überdies geschmälert, da für 2008 und 2009 jeweils 0,5 \% davon auf ein höheres Volumen für das Leistungsentgelt entfallen sollten - eine Zahlung, die nicht allen Beschäftigten zugute käme.

In der dritten Verhandlungsrunde am 11./12. Februar besserten die Arbeitgeber ihr Angebot nicht nach. Darauf reagierten die Gewerkschaften mit einer massiven Warnstreikwelle vom 14. bis 22.2., die jeden Tag einen anderen inhaltlichen Schwerpunkt hatte, von Krankenhäusern über Verwaltungen, Kindertagesstätten bis hin zum Nahverkehr und der Ver- und Entsorgung, und alle Bundesländer einbezog. Es beteiligten sich rund 200.000 Beschäftigte. Nachdem auch die vierte Verhandlungsrunde am 25./26.2. ohne Ergebnis blieb, folgte eine zweite noch stärkere bundesweite Warnstreikwelle vom 4 . bis 6.3. mit rund 230.000 Beteiligten, die auch die Flughäfen mit einbezog. Die fünfte Verhandlungsrunde am 6./7.3. führte schließlich zum Scheitern, nachdem die Arbeitgeber eine Verbesserung ihres Angebots erneut von einer Verlängerung der Arbeitszeit abhängig gemacht und zu diesem Zweck auch die tariflichen Arbeitszeitbestimmungen zum 30.4.2008 gekündigt hatten.

Nach dem Scheitern der Verhandlungen riefen die Arbeitgeber die Schlichtung an, die unter Leitung des früheren badenwürttembergischen Ministerpräsidenten Lothar Späth (CDU) und des langjährigen Hannoveraner Oberbürgermeisters Herbert Schmalstieg (SPD) stattfand. Der Schlichtungsvorschlag, der mit den Stim- men von Lothar Späth und den Arbeitgebervertretern zustande kam, sah vor:

- eine Tariferhöhung um 4,0 \% im Westen zum 1.4. , im Osten zum 1.8.;

- eine weitere Anhebung um 2,0\% ab 1.1.2009;

- eine Einmalzahlung von $450 €$ zum April 2008 für die Entgeltgruppen 1-8 sowie $450 €$ für alle Beschäftigten im Juli 2009;

- Verlängerung der Arbeitszeit im Westen auf 39,5 Stunden.

Nach der Vorlage des Schlichtungsvorschlages nahmen die Tarifparteien am 29.3. die Verhandlungen wieder auf. Die Gewerkschaften lehnten den Schlichtungsvorschlag ab. Er biete auf die Laufzeit umgerechnet und unter Berücksichtigung der Arbeitszeitverlängerung nicht einmal einen Inflationsausgleich. Die angebotenen Einmalzahlungen seien zudem nicht tabellenwirksam. Für größere Teile des öffentlichen Dienstes würde daher im Jahr 2008 der Reallohnverlust fortgeschrieben. Die vorgeschlagene Verlängerung der Arbeitszeit werde zu Stelleneinsparungen führen. Dies sei bei der hohen Arbeitslosigkeit in Deutschland kontraproduktiv. Unter dem Druck eines drohenden Streiks gelang nach dreitägigen Verhandlungen am 31.3. eine Einigung.

\section{ERGEBNISSE}

Die Ergebnisse beziehen sich sowohl auf die Tarifentgelte als auch auf die Arbeitszeitbestimmungen. In einigen Punkten wurden auch Sonderegelungen für einzelne Bereiche des öffentlichen Dienstes getroffen:

Entgelt:

- vorab $50 €$ Erhöhung (das entspricht im Durchschnitt der Entgeltgruppen einer Erhöhung um 2,0 \%);

- Erhöhung der Tarifentgelte um 3,1 \% ab 1.1.2008; (in den Gemeinden Ost jeweils ab 1.4.2008);

- 2,8 \% Stufenerhöhung ab 1.1.2009;

- $225 €$ zusätzliche Einmalzahlung im Januar 2009;

- Erhöhung der Auszubildendenvergütung um $70 €$ in allen Ausbildungsjahren; - Laufzeit bis 31.12.2009.

\section{Ost/West-Niveau}

- Bund Ost: Vorziehen der Anpassung von 92,5 auf $100 \%$ des Westniveaus für die Ent- 
geltgruppen $10-15$ vom 1.1.2010 auf den 1.4.2008 (aus Abschluss 2003: Anpassung des Tarifniveaus der Entgeltgruppen 1-9 von 92,5/97\% (Bund/Gemeinden) auf $100 \%$ des Westniveaus ab 1.1.2008).

\section{Arbeitszeit}

- Gemeinden West: Verlängerung der Wochenarbeitszeit von 38,5 auf 39 Std. ab 1.7.2008, ansonsten unveränderte Wochenarbeitszeit Bund West und Ost: 39 Std., Gemeinden Ost: 40 Std.;

- Wegfall der Öffnungsklausel mit der Möglichkeit der Verlängerung der Arbeitszeit auf bis zu $40 \mathrm{Std}$./Woche durch landesbezirklichen Tarifvertrag und Anpassung der auf dieser Grundlage geschlossenen Tarifverträge an die neue Arbeitszeit-Regelung (abweichende Regelung für den Arbeitszeit-Tarifvertrag Niedersachsen);

- Verwendung von 2,5 Arbeitstagen für Zwecke der Vorbereitung und Qualifizierung im Erziehungsdienst.

Sonstiges:

- Hinwirken der Tarifparteien auf die befristete Übernahme Ausgebildeter für zwölf Monate, jedoch nicht bei Ausbildung über den Bedarf hinaus;

- Für die Bereiche Krankenhäuser, Nahverkehr und Versorgung wurden Sonderregelungen getroffen;

- Die bereits vereinbarten Änderungen und Ergänzungen zum TVöD werden zum 1.7.2008 in Kraft gesetzt.

Die ver.di-Bundestarifkommission (BTK) nahm am 31. März in Potsdam mit $64 \mathrm{zu}$ 25 Stimmen die Tarifeinigung an. Ver.di führte vom 1. bis 11.4. eine breit angelegte Mitgliederbefragung durch, die zur Diskussion des Tarifergebnisses und zugleich für eine intensive Mitgliederwerbung genutzt werden sollte. Bei dieser Befragung sprachen sich 76,5\% der Mitglieder für die Annahme des Tarifergebnisses aus.

Der Abschluss wurde von den Gewerkschaften des öffentlichen Dienstes sehr positiv bewertet. Insbesondere Struktur und Volumen der Entgelterhöhung wurden herausgestellt. Die Verlängerung der Arbeitszeit wurde als vertretbares Zugeständnis gewertet. Nach Auffassung von Bundesinnenminister Wolfgang Schäuble ist man an die Grenze des haushaltsmäßig und gesamtwirtschaftlich Machbaren gegangen. „Aber es galt, im gesamtstaatlichen Interesse dem Land lange und harte Arbeitskämpfe zu ersparen." (Erklärung vom
31.3.2008); ähnlich die kommunalen Arbeitgeber, die die Einigung als „schmerzhaften Kompromiss" ansahen, der nur zu rechtfertigen gewesen sei, um einen Streik mit all seinen wirtschaftlichen und sozialen Beeinträchtigungen zu vermeiden (Presseerklärung vom 31.3.2008).

\subsection{CHEMISCHE INDUSTRIE}

In der chemischen Industrie konnte wie auch in der Stahlindustrie die vergleichsweise positive Entwicklung der vergangenen Jahre fortgesetzt werden. Zusätzlich zur Vereinbarung einer Tarifanhebung, die für 2008 deutlich über der erwartbaren Preissteigerungsrate liegt, wurde ein tarifpolitisches Paket „Lebensarbeitszeit und Demografie " geschnürt, das bereits bestehende tarifliche Regelungen zusammenfasst und um betriebliche Demografiefonds ergänzt.

\section{AUSGANGSSITUATION UND FORDERUNG}

Die chemische Industrie lag in dieser Tarifrunde zeitlich gesehen an dritter Stelle. Für den Bereich der Industrie konzentrierte sich nach dem Stahlabschluss die tarifpolitische Aufmerksamkeit auf diesen Wirtschaftszweig. Die Entgeltabkommen liefen regional unterschiedlich zwischen Februar und April 2008 aus. Wie üblich veröffentlichte der IG BCE-Hauptvorstand bereits im Vorfeld der Tarifrunde, dieses Mal am 12.12.2007, seine Forderungsempfehlung. Vor dem Hintergrund der stark steigenden Preisentwicklung sollte ein Tarifabschluss angestrebt werden, der - neben dem Ausgleich der Preissteigerung - die Produktivitätsentwicklung der chemischen Industrie berücksichtigt, sodass eine reale Einkommenserhöhung erreicht wird. Den Rahmen für eine Forderung bezifferte der Hauptvorstand zwischen 6,5\% und $7 \%$. Damit wurde - anders als in den Vorjahren - die Entgeltforderung wieder beziffert. Darüber hinaus wurde für den zum Jahresende 2008 auslaufenden Tarifvertrag „Zukunft durch Ausbildung" ein AnschlussTarifvertrag zur Sicherstellung und Weiterentwicklung des Ausbildungsplatzniveaus gefordert. Aufgrund der demografischen Entwicklung forderte die IG BCE außerdem den Abschluss eines Tarifvertrages über flexible Instrumente zur Gestaltung der Lebensarbeitszeit. Darin sollten u.a. die Rahmenbedingungen für eine alterns- und altersgerechte Arbeitsgestaltung und Möglichkeiten des flexiblen Übergangs in die Altersrente geregelt werden. Im Februar 2008 wurden die Forderungen endgültig beschlossen. Die Entgeltforderung wurde auf $7,0 \%$ festgelegt.

\section{VERHANDLUNGEN UND ERGEBNIS}

Wie in früheren Jahren wurde auch in dieser Tarifrunde zunächst auf regionaler Ebene verhandelt. Ab der zweiten Runde waren zentrale Verhandlungen geplant. Die regionalen Verhandlungen begannen am 26.2. in Hessen, es folgten RheinlandPfalz (27.2.), Nordrhein (28.2.), Bayern (3.3.), Baden-Württemberg (4.3.), Berlin West (7.3.), Saarland (10.3.), Westfalen (11.3.), Niedersachsen (13.3.) und Schleswig-Holstein/Hamburg (14.3.). Im Mittelpunkt stand der ausführliche Austausch über die ökonomischen Rahmenbedingungen, insbesondere in der chemischen Industrie.

Am 1.4. fand die erste Verhandlung auf Bundesebene statt. Erwartungsgemäß legten die Arbeitgeber zur Entgeltforderung noch kein Angebot vor. Fortschritte wurden dagegen nach Angaben der IG BCE in der Ausbildungsfrage und auch beim Thema flexibler Übergang in die Rente gemacht. Die Gespräche wurden auf den 15. und 16.4. vertagt. Anders als noch im Vorjahr gab es offenbar keine ernsthaften Konflikte, sodass diesmal eine größere Mobilisierung der Belegschaften unterblieb. Bereits in der zweiten Runde konnte dann am 16.2. ein Abschluss erzielt werden, der folgende Bestandteile beinhaltete:

Entgelt:

- Anhebung der Entgelte regional unterschiedlich ab März, April oder Mai 2008 für jeweils 13 Monate um 4,4 \%;

- Stufenanhebung um weitere 3,3 \% für jeweils zwölf Monate;

- zusätzliche Einmalzahlung in Höhe von insgesamt 7,0/7,8/9,1 \% eines Monatsentgelts für Beschäftigte in Normal-/teil-/vollkontinuierlicher Schicht zahlbar spätestens bis Ende Juli 2008 (dies entspricht 0,5 \% für den Zeitraum von 13 Monaten);

- Öffnungsklausel: Kürzung oder Wegfall des Betrages ist aus wirtschaftlichen Gründen möglich.

\section{Ausbildung:}

- Ausbildungsvergütungen: Erhöhung der regional unterschiedlichen Beträge um 
$34 € /$ Monat für 13 Monate und $26 € /$ Monat für weitere zwölf Monate;

- Verlängerung des Tarifvertrages „Zukunft durch Ausbildung" und Konkretisierung für die Jahre 2009 und 2010;

- Festschreibung des Ausbildungsplatzangebots von insgesamt 16.800 Ausbildungsplätzen für die Jahre 2009 und 2010;

- Ausbildungsplatzzahlen nach 2010 werden im Rahmen künftiger Verhandlungen festgelegt.

\section{Tarifvertrag „Lebensarbeitszeit und Demografie“:}

Mit diesem Tarifvertrag wird eine ganze Reihe von Regelungen beschrieben und zusammengefasst, die dem Themenkomplex zugeordnet werden können. Die Präambel geht auf die Bedeutung des demografischen Wandels ein und formuliert als Zielvorstellung die Förderung einer „nachhaltigen und vorausschauenden Personalpolitik“, die Anreize für eine längere Beschäftigung setzen soll. Als Elemente einer „Chemieformel zum demografischen Wandel“" werden genannt:

- Durchführung einer Demografieanalyse (Alters- und Qualifikationsstrukturen); - Maßnahmen zur alters- und gesundheitsgerechten Gestaltung des Arbeitsprozesses mit dem Ziel der Verbesserung der Beschäftigungs- und Leistungsfähigkeit; - Maßnahmen zur Qualifizierung während des gesamten Arbeitslebens;

- Maßnahmen der (Eigen-)Vorsorge und Nutzung verschiedener Instrumente für gleitende Übergänge zwischen Bildungs-, Arbeits- und Ruhestandsphase.

Die Demografieanalyse des Betriebes bzw. Unternehmens soll bis spätestens Ende 2009 durchgeführt und dann fortgeschrieben werden. Sie dient als Basis für die Durchführung konkreter Maßnahmen.

Auf betrieblicher Ebene wird ein Demografiefonds eingerichtet, in den der Arbeitgeber ab 2010 jährlich einen Betrag von $300 €$ je Beschäftigten einzahlt. Dieser Betrag wird ab 2011 um den jeweiligen Prozentsatz der Einkommenserhöhung des Vorjahres erhöht (Tarifdynamisierung). Im Rahmen einer freiwilligen Betriebsvereinbarung kann der Demografiebetrag (nur) für folgende Zwecke verwendet werden: Langzeitkonten, Altersteilzeit, Teilrente, Berufsunfähigkeitszusatzversicherung und tarifliche Altersvorsorge. Erfolgt bis Ende 2009 keine Einigung zwischen Arbeitgeber und Betriebsrat, muss der Arbeitgeber in Betrieben mit bis zu 200 Beschäftigten den Demografiebetrag für die tarifliche Altersvorsorge, in größeren Betrieben in Form eines Langzeitkontos zur Verfügung stellen. Die bisherigen tariflichen Regelungen zu Langzeitkonten und zur Arbeitsgestaltung gehen in veränderter Form ebenso wie die Tarifverträge zur Qualifizierung sowie zur Altersteilzeit in den neuen Tarifvertrag über.

Für die ostdeutsche chemische Industrie einigten sich die Tarifvertragsparteien am 27.5. auf einen Abschluss, nach dem die Entgelte, wie im Bundesgebiet West, um 4,4 \% ab Mai 2008 und um weitere 3,3\% ab Juni 2009 erhöht werden. Auch die zusätzliche Einmalzahlung wurde übernommen. Die Tarifparteien einigten sich ferner auf eine Niveauangleichung an Berlin-West in Höhe von 2,0 und 1,98 \%, jeweils zahlbar ab Oktober 2008 und 2009. Damit wird die bereits 2002 im Grundsatz vereinbarte Anpassung der Osttarife an das Westniveau erreicht. Der Entgeltaufbau der Gruppen E 5 bis 8 wurde weiterentwickelt, dadurch steigen die Endstufen ab Oktober 2009 zwischen 0,6 und 2,6\%. Außerdem wurde vereinbart, dass in den Jahren 2008 und 2009 jeweils 700 Ausbildungsplätze angeboten werden. Der Tarifvertrag „Demografie und Lebensarbeitszeit" wird auch im Bundesgebiet Ost umgesetzt.

Die IG BCE wertete den Tarifabschluss insgesamt als „zukunftsweisend“ und als „tarifpolitischen Meilenstein“. Auch die Chemie-Arbeitgeber beurteilten die Regelungen zur Ausbildung sowie zur Demografie und Lebensarbeitszeit als „wegweisend". Mit den Tarifanhebungen sei man an die „Belastungsgrenze“ gegangen. Die lange Laufzeit von zwei Jahren und die Flexibilisierung der Einmalzahlung verschaffe den Unternehmen mehr Planungssicherheit (BAVC-Presseinformation vom 16.4. 2008). BDA-Präsident Hundt warnte, wie bereits beim Stahlabschluss, dass die Einigung im Hinblick auf die Höhe der Belastung kein Maßstab für die gesamte Wirtschaft sein könne.

\subsection{EINZELHANDEL}

Der Tarifkonflikt des Einzelhandels schwelte seit dem Frühjahr 2007. Kernpunkt der Auseinandersetzung war die Forderung der Arbeitgeberverbände, die tariflich geregelten Spätarbeitszuschläge zu streichen, nachdem die Ladenöffnungszeiten drastisch verlängert worden waren. Auf diese Weise sollten die Personalkosten in den Spätöffnungszeiten deutlich verringert und damit die neuen Öffnungszeiten (überhaupt erst) rentabel gemacht werden. Ver.di lehnte die Forderung vehement $\mathrm{ab}$ und versuchte mit rund 6.500 Streik- und Warnstreikaktionen einen Neuabschluss der gekündigten Manteltarifverträge sowie neue Lohn- und Gehaltstarifverträge durchzusetzen.

Nach 15 Monaten harter Auseinandersetzungen gelang am 10.7. in Baden-Württemberg ein erster Abschluss. Dieser sieht u.a. eine Pauschalzahlung von insgesamt $400 €$ für den Zeitraum April 2007 bis März 2008 sowie eine Erhöhung der Vergütungen von 3,0 \% ab April 2008 vor. Die Laufzeit endet zum 31.03.09. Des Weiteren wird der Manteltarifvertrag rückwirkend zum 1. Januar 2007 wieder in Kraft gesetzt. Die Spätarbeitszuschläge von Montag bis Freitag zwischen $18.30 \mathrm{~h}$ und $20 \mathrm{~h}$ in Höhe von $20 \%$ bleiben unverändert erhalten. Der Zuschlag von $20 \%$ für spätöffnungsbedingte Arbeit wird an Samstagen zukünftig in der Zeit von 18.30 bis $20.00 \mathrm{~h}$ fällig (bisher ab $14.30 \mathrm{~h}$ ). Als Ausgleich erhalten die Beschäftigten in den Jahren 2009 und 2010 jeweils $150 €$, wahlweise zur Altersvorsorge, als Gutschrift auf einem Langzeitkonto oder in Form eines Warengutscheins. Auf Basis dieses Abschlusses konnte dann auch in Nordrhein-Westfalen und im Saarland eine Einigung erzielt werden. Die stellvertretende Ver.di-Vorsitzende Margret Mönig-Raane wertete dies als „beachtlichen Erfolg, den sich die Kolleginnen und Kollegen mit ihren Streiks erkämpft haben“.

\section{Ausblick}

Die Tarifrunde 2008 ist noch nicht abgeschlossen: Offen waren zum Redaktionsschluss dieses Berichts noch die Verhandlungen im Bankgewerbe und noch nicht begonnen hat die diesjährige Tarifrunde in der Metall- und Elektroindustrie. Die Verträge laufen dort Ende Oktober aus. Auch in einigen kleineren Tarifbereichen wird noch verhandelt. Ende des Jahres läuft dann der Entgelttarifvertrag im öffentlichen Dienst-Bereich der Länder aus. 


\section{ITERATUR}

Bispinck, R./WSI-Tarifarchiv (2006): Tarifpolitischer Jahresbericht 2005, Düsseldorf, Januar

Bispinck, R./WSI-Tarifarchiv (2008): Tarifpolitischer Jahresbericht 2007: Aufwärtstrend bei Tarifabschlüssen - Konflikte um Beschäftigungssicherung und Tarifstandards, in: WSI-Informationen zur Tarifpolitik, Düsseldorf, Januar

Bundesministerium für Wirtschaft und Technologie (2008): Jahreswirtschaftsbericht 2008 - Kurs halten!, Berlin

IG Metall (2007): Tarifrunde 2008 in der Eisen- und Stahlindustrie: Sozialökonomische Rahmenbedingungen, Wirtschaftspolitische Informationen 10

Institut für Makroökonomie und Konjunkturforschung (IMK) (2007): Der Aufschwung geht weiter, Frühjahrsprognose des IMK für 2007, IMK-

Report 19
Meerkamp, A. (2008): Neugestaltung des Tarifrechts im öffentlichen Dienst, in: Bispinck, R. (Hrsg.): Verteilungskämpfe und Modernisierung. Aktuelle Entwicklungen in der Tarifpolitik, Hamburg, S. 109-122 Sachverständigenrat zur Begutachtung der gesamtwirtschaftlichen Entwicklung (SVR) (2007): Jahresgutachten 2007/08 - Das Erreichte nicht verspielen, Wiesbaden

Vereinigung der kommunalen Arbeitgeberverbände (VKA) (2007):

10 Punkte zur Tarifrunde 2008, Mannheim, 16. November

Vereinte Dienstleistungsgewerkschaft (ver.di) (2007): Tarifbewegung 2007/2008, Tarifrunde 2008 öffentlicher Dienst, Berlin o. J. 